\title{
Prototype of Intelligent Data Management System for Computer Animation (iMCA)
}

\author{
Hui Liang ${ }^{1}$, Fenglong $\mathrm{Wu}^{1}$, Jian Chang ${ }^{2}$, Meili Wang ${ }^{3 *}$ \\ ${ }^{1}$ Zhengzhou University of Light Industry, China \\ ${ }^{2}$ Bournemouth University, Poole, UK \\ ${ }^{3}$ Northwest Agricultural \& Forestry University, China \\ meili_w@nwsuaf.edu.cn
}

\begin{abstract}
In recent years, one of the most noticeable issues of current animation production is the challenge from the exponential growth of animation data known as an increasingly data-intensive process. There are obvious gaps between the animation production needs and research development, which call for novel design and new technology to tackle the emerging challenge of handling huge amounts of data. "iMCA" is designed to develop intelligent data management solution with the capability to handle massive and hyper type animation asset and analyze/summarize information for reuse of data to facilitate human creativity providing innovative interaction to allow the manipulation of massive animation data.
\end{abstract}

Keywords: Prototype, Intelligent Data Management, Animation Data Asset.

\section{Introduction}

Computer animation has been booming and prospering for decades. Now, computer animation production is an increasingly data intensive process. One of the significant changes faced by animation industry is the evolution of computer animation data [1]. The animation data management strategy of an animation company revolves around the creation, presentation, storage, retrieval and reuse of its valuable animation data assets, which is playing an important role in CG production and video game industry. The demands for modern technologies to boost the data processing are urgent.

However, even though advanced theories, methods, and tools help a lot for managing animation data, the process of animation asset to be maintained, retrieved and reused is still a tedious work and can also be labor-intensive. Its inherent features of complexity throw out challenges to current animation producers: how can we develop intelligent solutions with the capability to handle huge amounts of data and analyze/summarize information to facilitate human creativity?

Targeting this "hot issue" to current animation practitioners and researchers, a prototype of intelligent animation data management (iMCA) is proposed in this paper for

${ }^{*}$ Corresponding author 
the purpose of facilitating the storage, organization, retrieval, utilization and reuse of digital animation data assets. Semantic and ontological analysis is leveraged to understand and describe the complexity of animation data. Domain-specific ontology and an ontology-based animation data retrieval system is developed in the context of Chinese traditional shadow play art, which is used as a usage example to provide guidance in semantic animation data representation and retrieval. To provide seamless and scalable access to distributed resources of this data system, mobile app is designed to link the mobile clients to distributed servers, which allows access to animation data to remove the geographical constraints.

The rest of the paper is structured in four parts: Section 2 briefly reviews the related research works. Section 3 proposes a semantic model using ontological method to describe animation data asset. Section 4 presents the prototype of intelligent data management solution "iMCA" and Section 5 concludes this paper.

\section{$2 \quad$ Related Work}

Given the booming of the global computer animation industry in decades, more challenges arise in the extant animation production, especially the way we manage the data. There is no doubt that the animation industry is currently experiencing an accelerating increase of the constant and speedy generation of hybrid animation data. According to Desai's study, the animation data generated during the last few years has exceeded the total of the past [2]. Also, the animation data exists at almost all stages of the animation production in a wide variety of forms - text files, audio files, video files, texture files, graphic files, 3D model files, motion files, scene files, and many other types. And furthermore, within each of these types, the animation file may even be in different format. Taking the 3D model of a cartoon character for example, the 3D model file could be the format of *.ma or *.mb for MAYA, or *.max for 3DMax. The digital assets are expected to be stored and indexed in a fashion to be easily maintained, retrieved and reused.

A number of applications attempt to address some of the challenges of managing the animation data for the purpose of promoting animation production. Shotgun [3], Alienbrain [4] and TACTIC [5] are used by professional companies, which facilitates the monitoring of the pipeline and the management of data asset. However, the ability of current solutions is less than satisfactory: Alienbrain is similar to a kind of file storing and sharing system which lacks the functions of efficient information extracting and data reusing. Shotgun and TACTIC are commercial CG production toolsets, which mainly focus on production tracking rather than intelligent data managing.

To promote the reusability and accessibility of vast animation assets, text-based data retrieval is commonly used in current animation data management, which indexes the metadata such as keywords and tags associated with the annotated animation files [6]. However, the input of keywords or metadata can be labor-intensive and time consuming, and even the entered information may not describe the desired animation data properly, which is still a grand challenging and interesting problem in the field of multimedia information retrieval [7]. Contrastively, the content-based retrieval facili- 
tates the searching of the animation data in a higher efficient and more user-friendly way, which analyzes and matches the content of the animation files rather than the metadata, e.g., the colors, contour profile, textures, or any other content information derived from the animation files [8]. But, both the traditional text-based and the content-based media data retrieval only depend on similarity matching of the textual or physical information [9]. The internal semantic information of the animation data is ignored. If the text or matching content does not exist, the retrieval cannot be executed. It has been proved that the semantic gap between the low level description and the high level semantic interpretation of multimedia objects does exist in the field of data management [10].

In recent years, being able to reflect users' query intent and consider the semantic information, semantics and ontology have begun to be used to describe the semantics of animation assets and data retrieval, which could reduce the semantic gap between low-level features and high-level semantics to achieve a better retrieval and to promote reusability [11].

Originated in philosophy, the term of ontology is an explicit formal specification of conceptualization that is used as an effective tool to describe general concepts of entities, as well as their properties, relationships and constraints [12, 13]. Taking the advantage of the establishment of common vocabulary and semantic term interpretation, the concept of ontology entered information science as a formal system for representing domain knowledge and their related linguistic realizations required by different applications that cover various fields, from knowledge engineering to software engineering $[14,15]$.

By defining semantic concepts, inferring usage rules and combining the semantic concepts and relations, we can leverage semantic and ontological analysis to understand and describe the complexity of animation data assets. Previous research on semantic data representation and retrieval provides us appealing solutions to construct a systematic and standardized model to describe animation data assets at a highly abstract and semantic level. Researchers in the AIM@SHAPE Network of Excellence project firstly use the semantic approach to interpret multimedia object for the purpose of facilitating animation file retrieval and reuse [16]. 3D model Search Engine [17] and Google 3D Warehouse [18] are also well-known semantic search engines for providing 3D content accessing. Ontology for Media Resources [19] and the Core Ontology for Multimedia (COMM) [20] are two specified ontologies developed to describe media data (e.g., images, 3D models, audio and video files) based on the standard models for data interchange (e.g., RDF, RDFS and OWL).

The concept of semantic and ontology is also incorporated into the description of complex animation content, for example, the virtual environment and games. The concept of annotated environment is proposed by Thalmann et al, which is modeled by ontology description at a high semantic level including the environment structures, entities' behavior and domain knowledge [21]. Some studies, for example, NiMMiT (Notation for MultiModal interaction Techniques) [22] and SCM (Semantic Content Model) [23] mainly focuses on the high-level description of 3D scenes. There are also some ontological applications in the domain of augmented reality and computer 
games. In Ruminski's research, a contextual augmented reality environment is provided to describe the virtual world using three kinds of elements: the trackables, content objects and interface [24]. Ontology is also used to describe games characteristics, properties and design process, to set rules to represent game logics and to construct ontological framework to develop and employ game-based training etc. [25-27].

Ontology-based semantic animation data description and management acts as an appropriate way to represent structured animation asset knowledge bases and advances in bridging the semantic gap, which provides us suitable technique on the animation assets management to facilitate data storage, organization, retrieval, reuse and repurposing. We consider leveraging semantic and ontological analysis to model animation data in a systematic and standardized way and then develop prototype of intelligent data management solution with the capability to handle huge amounts of data and to analyze information for reuse of data to facilitate human creativity.

\section{Semantic and Ontological Analysis of Animation Data}

\subsection{Semantic Model of Animation Assets}

Targeting different animation topics and depending on the specific application fields, the contents of the generated digital assets also vary. During the process of animation production, the digital assets are arranged in data repositories, which are multimodal and include a variety of data types - audio, video, 2D image/textual, 3D models, motion files, scene files etc. From a semantic perspective, the repository could be abstracted and analyzed in a systematic and standard way. In this session, a semantic model is proposed first to describe the animation data assets to provide a clear understanding of animation data, which is abstracted and analyzed with several sub-layers.

As illustrated in Fig. 1, the semantic model could be decomposed into four layers in the context of 3D animation production: Geometry, Structure, Appearance and Logic, from the basic geometry shape as the start to the logic meaning in the end. Geometry layer is the presentation of basic graphic elements. 2D content consists of simple metadata, such as points, lines, text, which further contributes to 3D content. 3D content provides low-level description of single model's geometric information and physical features, such as spheres, cones and other simple separated 3D objects. Structure layer is the combination of separated objects. Within this layer, single objects are combined into a complex one, for example, a table, which could be decomposed into simple objects, such as boxes etc. And the compounded object could also be integrated into other complex object to form a more complex model or as a part of a virtual scene. Appearance layer targets the appearance information including lighting, texture, color and transparency etc., which transforms the art design into working reality. Logic layer describes the contents' functions and properties from the aspect of logic, including the story plot, culture background and personality etc. 


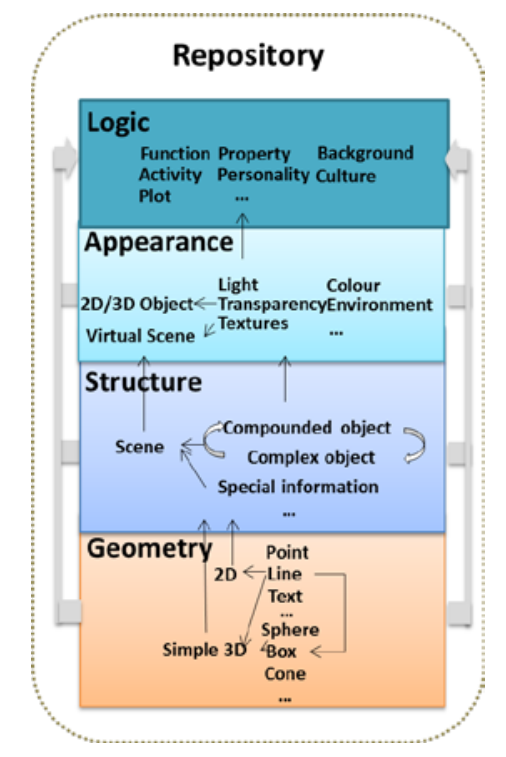

Fig.1. Semantic model to describe the animation data assets

Fig. 2 presents a usage example of the proposed semantic model, which describes the 3D model of a famous character - "Qin Shi Huang” in Chinese shadow play, who is the king of Qin Dynasty and China's first emperor. Mapped to the Geometry layer in the semantic model, the shape of the character is a composition of basic description of graphic elements, which provides low-level description of single model's geometric information and physical features, such as spheres, cones and other simple separated 3D objects. In the context of traditional Chinese shadow play, Chinese shadow puppet is mainly made of donkey's hide. A shadow puppet consists of several parts and its joints are connected by threads. When playing, puppeteers use their hands to manipulate the movement of the character through sticks attached on the different parts of the puppet. These different parts of the puppet are mapped to the Structure layer. Added with the appearance information mapped to the Appearance layer, such as color, pattern or accessories, a complete shadow play character is created. Further, provided with the information about story plot, culture background and personality, this character could carry rich traditional culture contents - the Logic layer. As a higher level of expressiveness, it represents a most famous Emperor in Chinese history. 


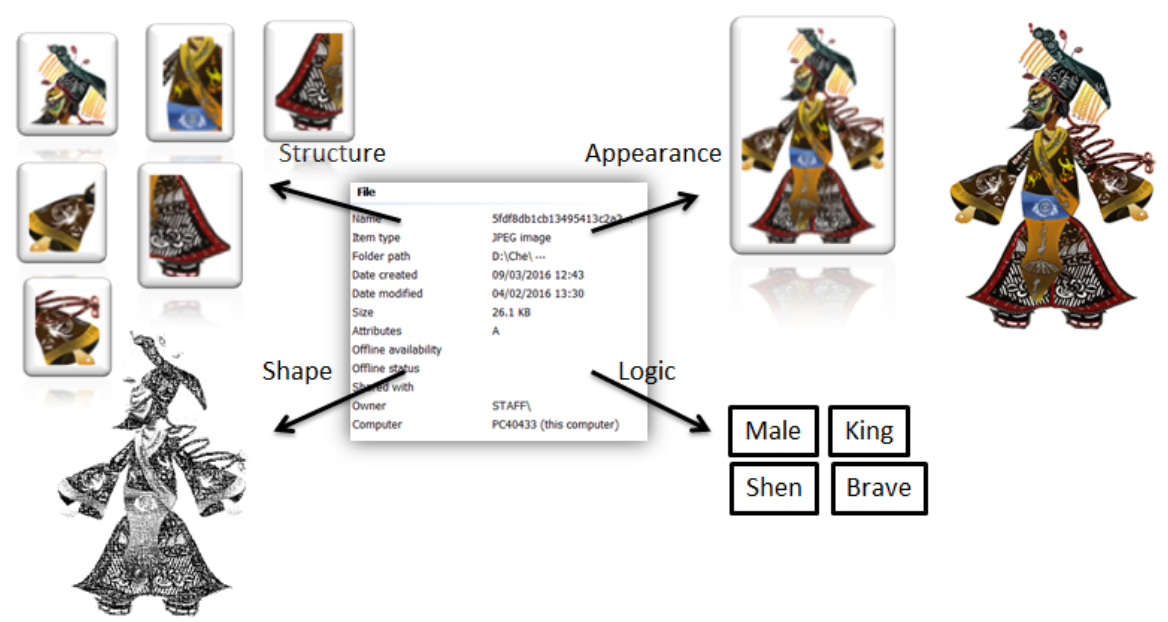

Fig.2. Usage example of semantic model-“Qin Shi Huang”, the king of Qin Dynasty in China

\subsection{Chinese Shadow Animation Assets Ontology}

The proposed semantic model describes the animation data from the abstract high level, which guides the implementation of the domain specified ontology further. As a usage example, we used this ontology-based data management method in animating the performance of a Chinese traditional shadow play titled "The Emperor and the Assassin": a brave fighter named "Jing Ke" attempted to assassinate the king of Kingdom Qin -“Qin Shi Huang” to avert the imminent conquest of his home country by Kingdom Qin. After intense fighting in the palace, however, the assassination attempt failed, and "Jing Ke" was killed on the spot. The animation data asset used in this animation is presented by developing a domain-specific ontology: Chinese Shadow Animation Assets Ontology (CSAAO) as illustrated in Fig. 3, which is the ontological implementation of the proposed semantic model.

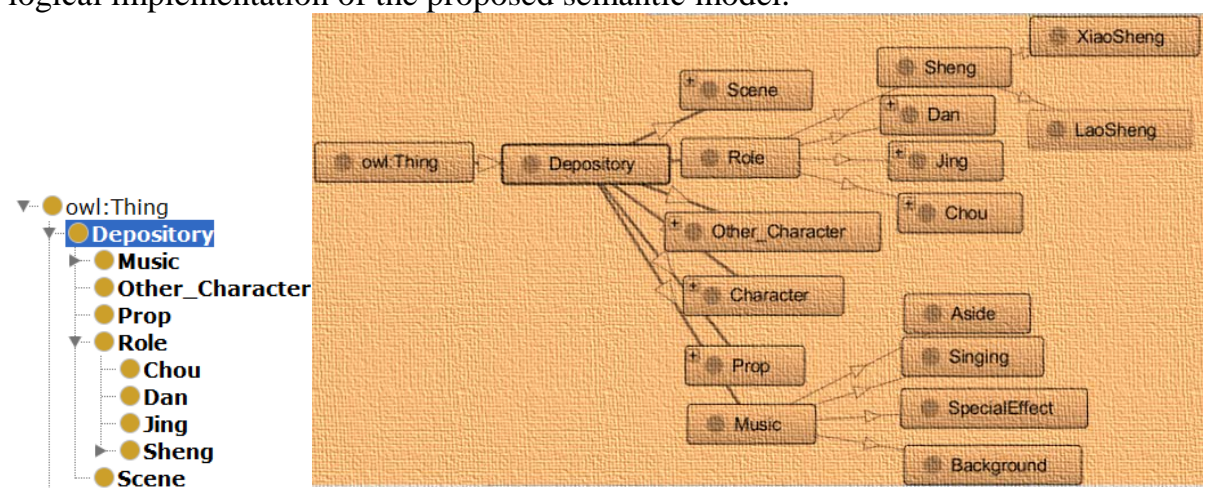

Fig.3. Diagram of Chinese Shadow Animation Assets Ontology (CSAAO) 
The main point in constructing ontology is to define a basis knowledge associated to the applied domain, a finite set of basic elementary relations description, and a set of functions operating. The basic variables then are used to label the type of an entity or an argument in a predicate.

Following composing the ontology basis is to construct ontology for a portion of the Chinese shadow play art domain covered in CSAAO:

R. Roles, defines the human characters of traditional Chinese shadow puppetry, which falls into four specific sub-types: Sheng (main male role), Dan (female role), Jing (painted-face and forceful male role), Chou (clown male role).

M. Music, defines melodies played by the accompaniment, which have standard melodies to indicate different art atmosphere. For instance, manban represents a slow tempo, yuanban represents standard or medium-fast tempo etc.

P. Props. On stage, characters interact with different objects to serve different purposes. For example, a piece of dark cloth is hung up to indicate a city wall.

S. Scene. Normally, a full-length shadow play consists with several key scenes. Each scene is a unit of action and follows the pattern of emotional progression. In a major scene, the beginning and end are often marked by a change of characters onstage or changing the stage set.

For instance, let us have the following predicates:

has_Prop_of (Roles:R, Props: P)

has_Music_of (Roles:R, Music: M)

has_Role_of (Scene: S, Roles:R)

Note that predicates has_Prop_of, has_Music_of and has_Role_of keep explicit polymorphism with respect to Roles. Using these predicates we are able to code, for instance, the information from the text "retrieval the props and music data asset used in the scene of the 'The Emperor and the Assassin"” as:

solution search has_Role_of (Scene:S, Roles:R)

->has_Music_of (Roles:R, Music: M)

and

->has_Prop_of (Roles:R, Props: P)

If we set S="The Emperor and the Assassin", then all the roles involved in this scene are returned according to the predicates of has_Role_of, and after that, all the props and music related with the retrieved roles are searched by referring the predicates of has_Music_of and has_Prop_of.

We also propose data properties which provide attribute descriptions for classes in the CSAAO. Taking the class of Role for example, the data properties of a character include "name”, "age”, "rank" and "personality” etc. Fig. 4 shows a usage example of the Role class defined in the ontology. 


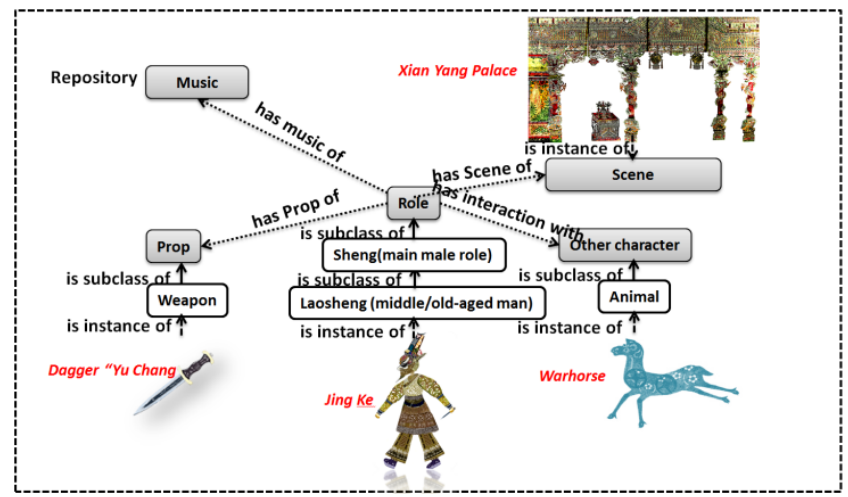

Fig.4. Usage example of CSAAO-“Jing Ke”

\section{Prototype of Intelligent Animation Data Management Solution "iMCA"}

A prototype of intelligent animation data management system is built upon a central repository developed on the base of the proposed ontology CSAAO that facilitates the storage, organization, retrieval, utilization and reuse of animation assets. The system architecture is shown in Fig. 5.

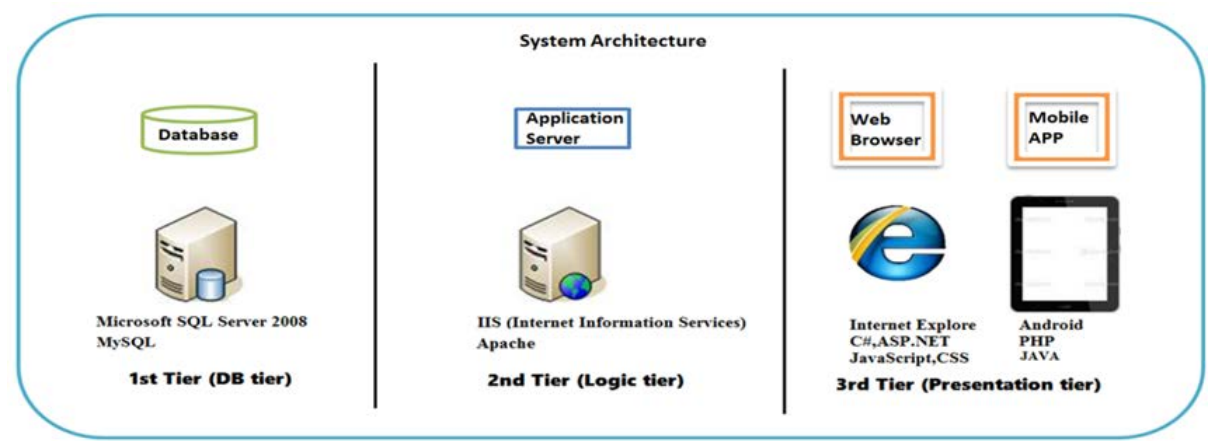

Fig.5. System architecture of "iMCA"

\subsection{Ontology-based Animation Assets Retrieval}

As shown in Fig. 6, we developed a visualization tool to demonstrate the concept of proposed ontology CSAAO, which leverages ontology to construct a systematic and standardized model to describe at a highly abstract and semantic level to provide a full view and understanding of the complex domain knowledge. Using structured terminology, ontological analysis could capture the core logic of complex system with natural language descriptions. This tool provides researchers with user-friendly and dynamic interaction: each colored circle encloses a subclass that fall under its super- 
class; users can click on any of the circles to zoom in or drag and drop them for interaction and check the entities' properties, relationships and constraints between them.

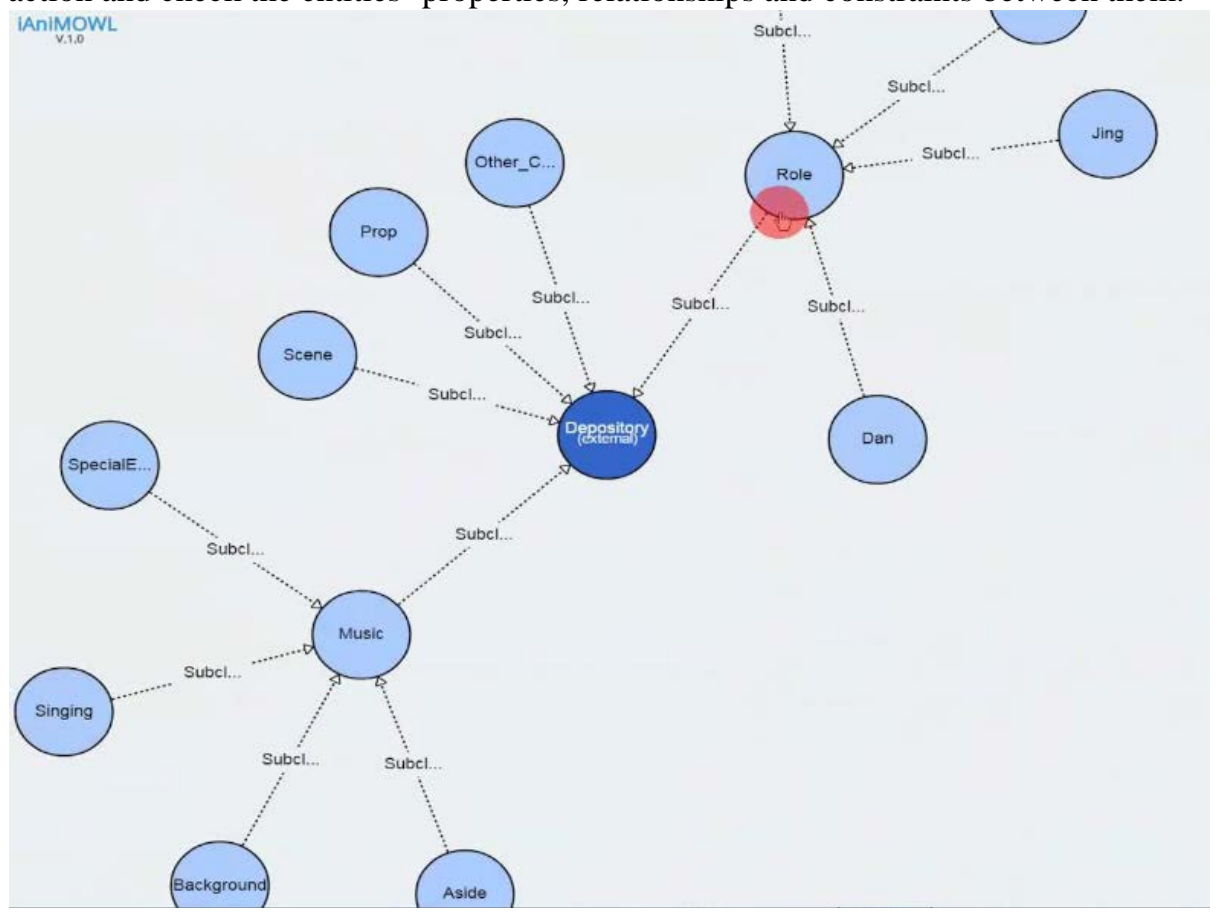

Fig.6. Interactive illustration of CSAAO

The ontology based "smart" digital assets management prototype provides seamless and scalable access to distributed animation resources. Animation data set interpret/display the information in a user-friendly manner. Assets Repository is a mixedtype animation database involving domain specified knowledge of traditional Chinese shadow play art using domain-specific information extraction, inference and rules to exploit the semantic metadata. This repository supports ontology-based retrieval, which improves searching performance by recognizing the animator's intent and contextual meaning of the digital assets in the context of Chinese traditional shadow play.

Through this ontology-based retrieval system which maps artist's intention on the data stored in the digital assets ontology, we can handle domain related query and provide more reasonable and relevant feedback to meet the artist's needs and to facilitate data reuse. 


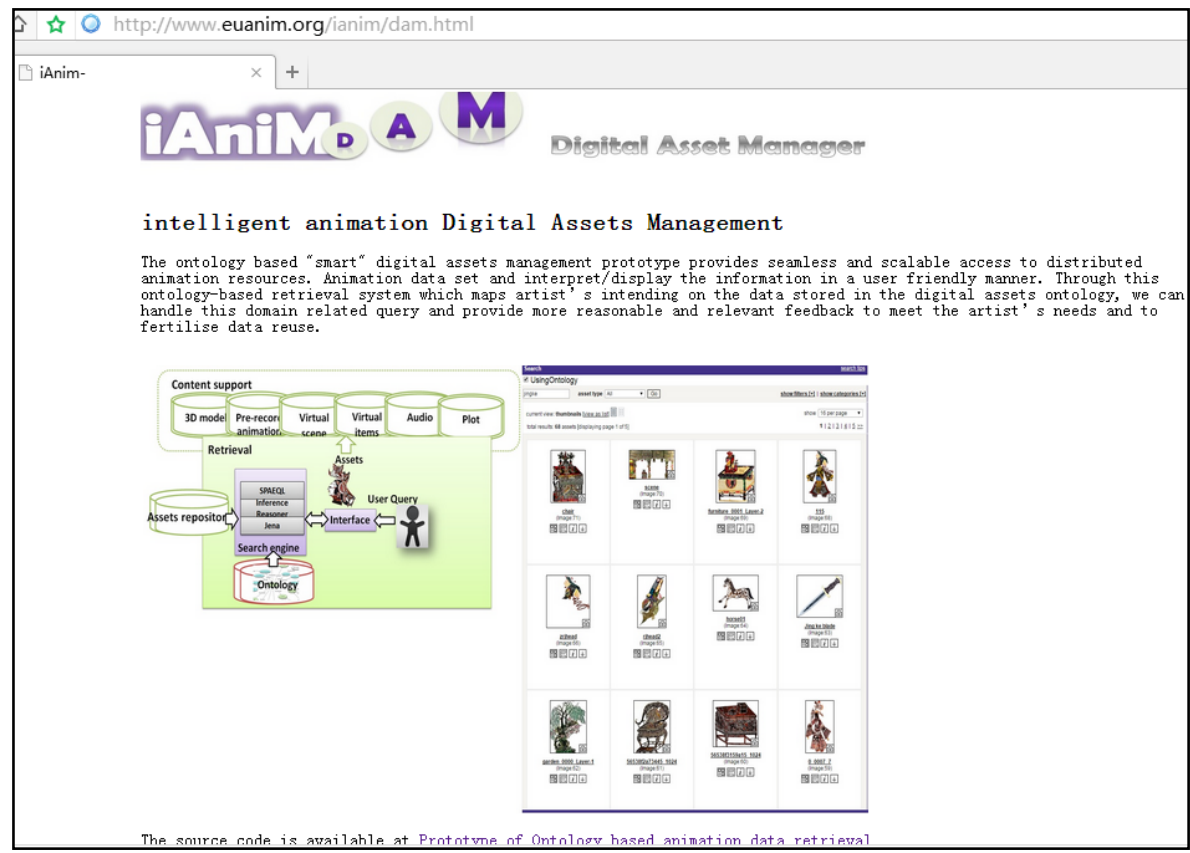

Fig.7. Retrieval system

As illustrated in Fig. 8 (a), when inputting the name of the character “Jing Ke", using traditional keywords matching, only the asset of character "Jing Ke" was retrieved. However, the best possibility is that there is still other animation asset may exist relating to "Jing Ke", such as his personalized weapon and his warhorse etc. This kind of asset is expected to provide more reasonable and relevant feedback to animators using ontological reasoning and referring through SPARQL queries. The result of ontological retrieval in Fig. 8 (b) shows that not only the digital character "Jing Ke" is retrieved, but also other assets related to the searching target are also retrieved from the knowledge base.

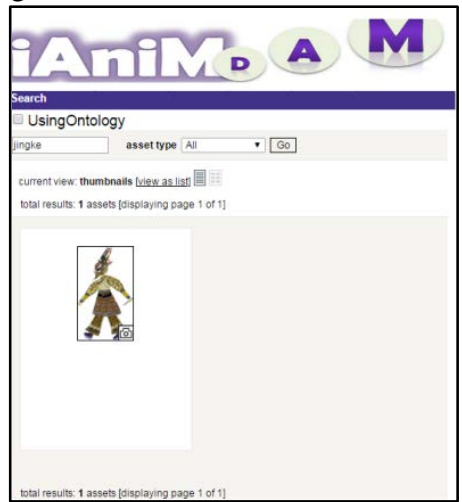

(a) 


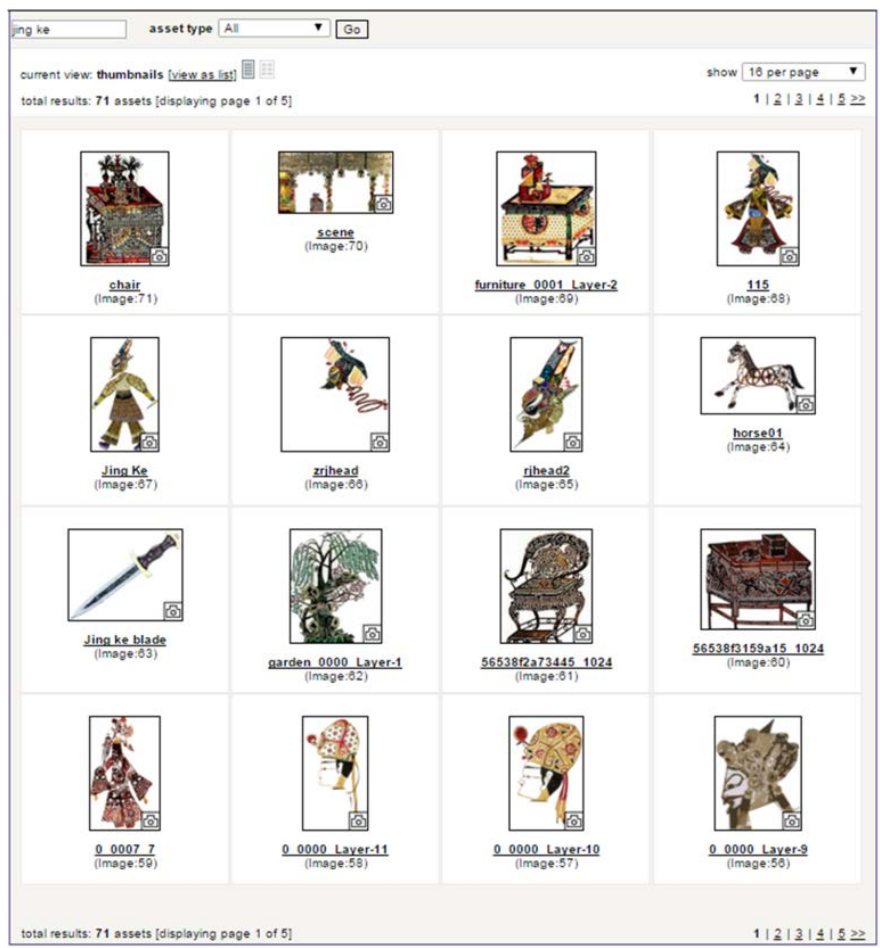

(b)

Fig.8. Interface of ontology based retrieval system

The following SPARQL Query Language is used as inputs: ty ?object WHERE \{ base: Jing_Ke? property ?object\}".

The query will return a result that contains the set of all properties and objects that is related to the character "Jing $\mathrm{Ke}$ ".

Pseudocode of using a Java framework for building Semantic Web - Jena API [46] for querying from database is described as in Table 1 below:

Table 1. Pseudocode for querying from database

// create a connection to animation asset database

DBConnection conn=new DBConnection(URL， USER，PASSWORD);

//use connection to construct model (MODEL_NAME) in the database:

ModelRDB m = ModelRDB.open(conn, MODEL_NAME);

//create shadow puppet assets ontology model spOntology

OntModel spOntology = ModelFactory.createOntologyModel();

//create OWLReasoner, and bind with spOntology 
Reasoner owlReasoner=ReasonerRegistry.getOWLReasoner();

Reasoner spReasoner=owlReasoner.bindSchema(spOntology);

//create inferring model

IModel iModel=ModelFactory. createIModel(spReasoner， imodel);

//When Query is ready; it will be passed to QueryEngine to execute querying

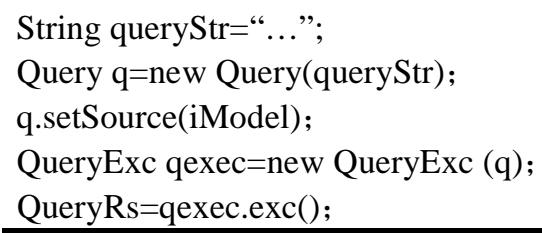

\subsection{Mobile App}

Taking the advantage of the more economical and accessible technology tools, digital media production has evolved to be a highly collaborative activity that involves teams of people working with digital resources in different locations. The concept of collaborative production can be seen through the four core ideas: Opening, Peering, Sharing and Acting globally.

For the purpose of exchanging information and knowledge among animation studios and collaborators, a novel mobile app is developed which allows a great dynamism in the creative process and speeds up production. The mobile App makes it simple to search, browse, and view media cross all projects from one place in the cloud - then share them with anyone on the project, anywhere in the world.

App lets the artist access, review, and gives feedback on all the versions (media) they track in the shadow play animation asset, which provides artists with the following functions:

- Browse media, play back animation clips, movies and playlists in all the animation projects, whether at desktop or on-the-go. As long as having an internet connection, users can review from anywhere on the mobile device. App makes review easy. Screenshots of the data asset list and reviewing 3D model are shown in Fig. 9 and Fig. 10 . 


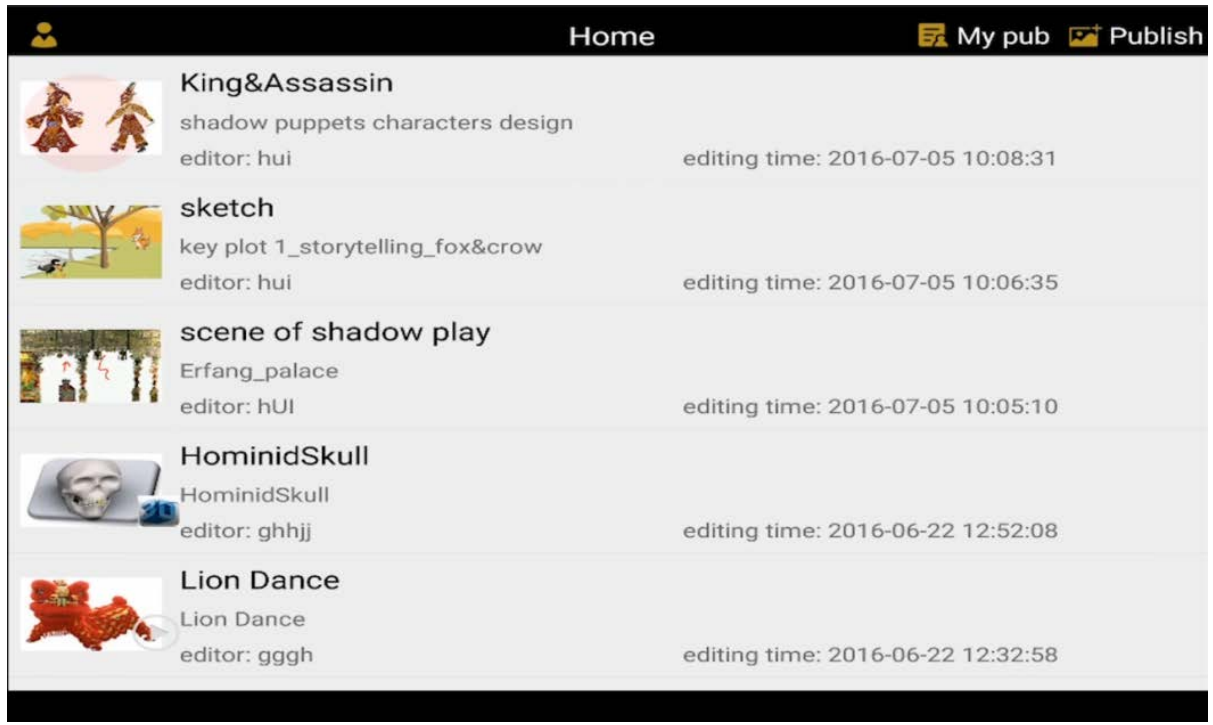

Fig.9. Screen shot of the asset list

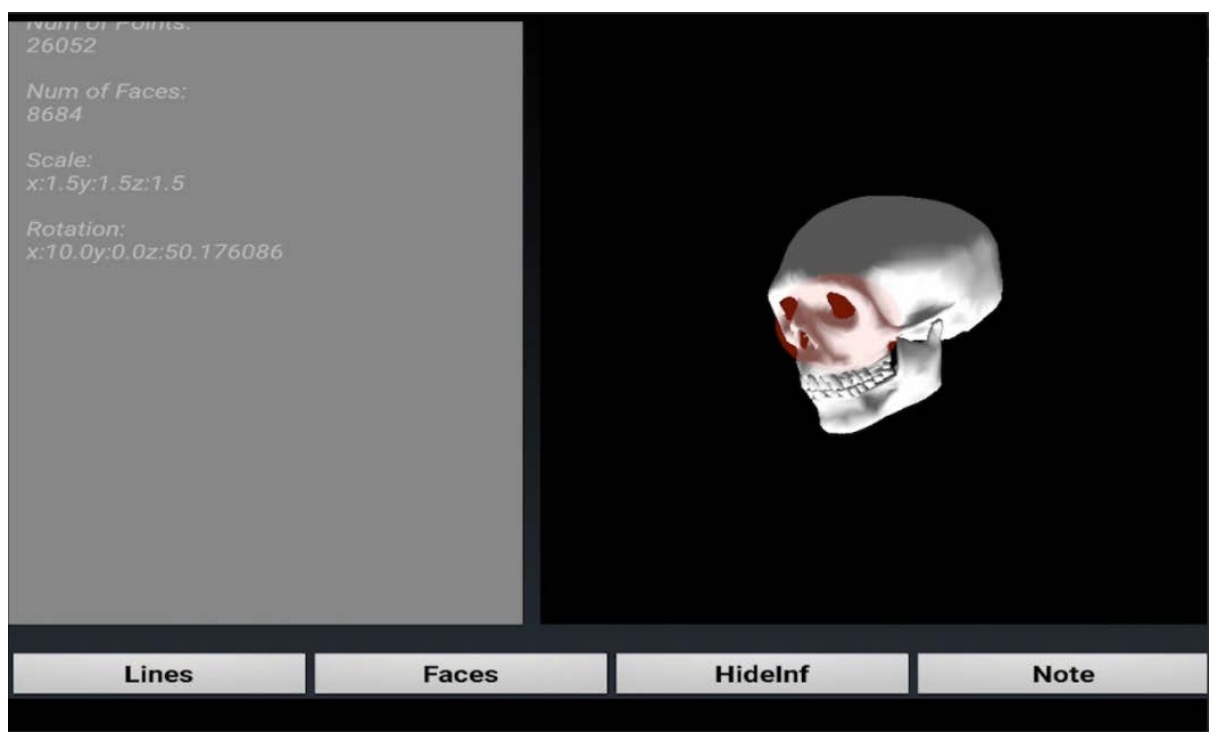

(a) Check detail by rotating and scaling 


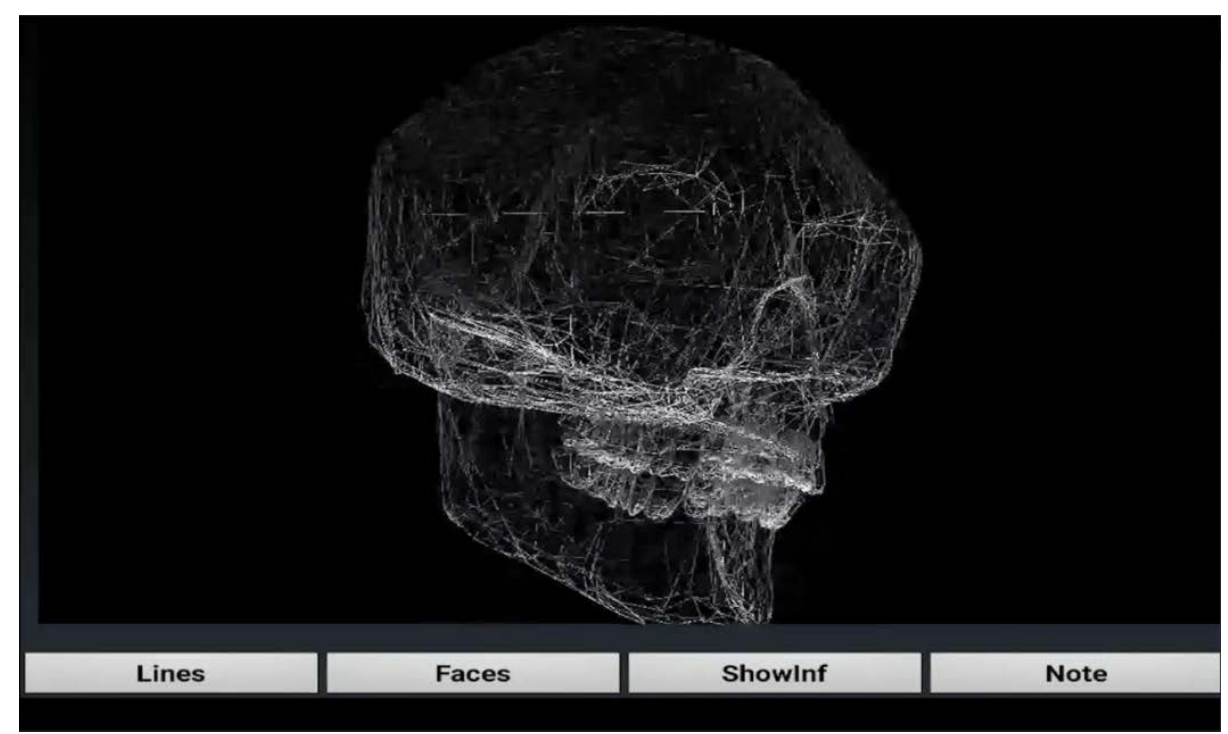

(b) Review wireframe model

Fig.10. Screen shot of reviewing asset

- Give feedback with comments, annotated frames, or camera images, add comments and draw right on top of media to pinpoint exactly where to find feedbacks. Artists receive a note with the annotated images detailing exactly what to do. Screen shots of providing annotation are illustrated in Fig. 11.

- See history on related versions and their notes. Every note and annotation is tied to the central repository as shown in Fig. 12. Feedback and creative direction coming out of a review session is stored in one place and all the involved people are notified, so everyone is on the same page at all times. 


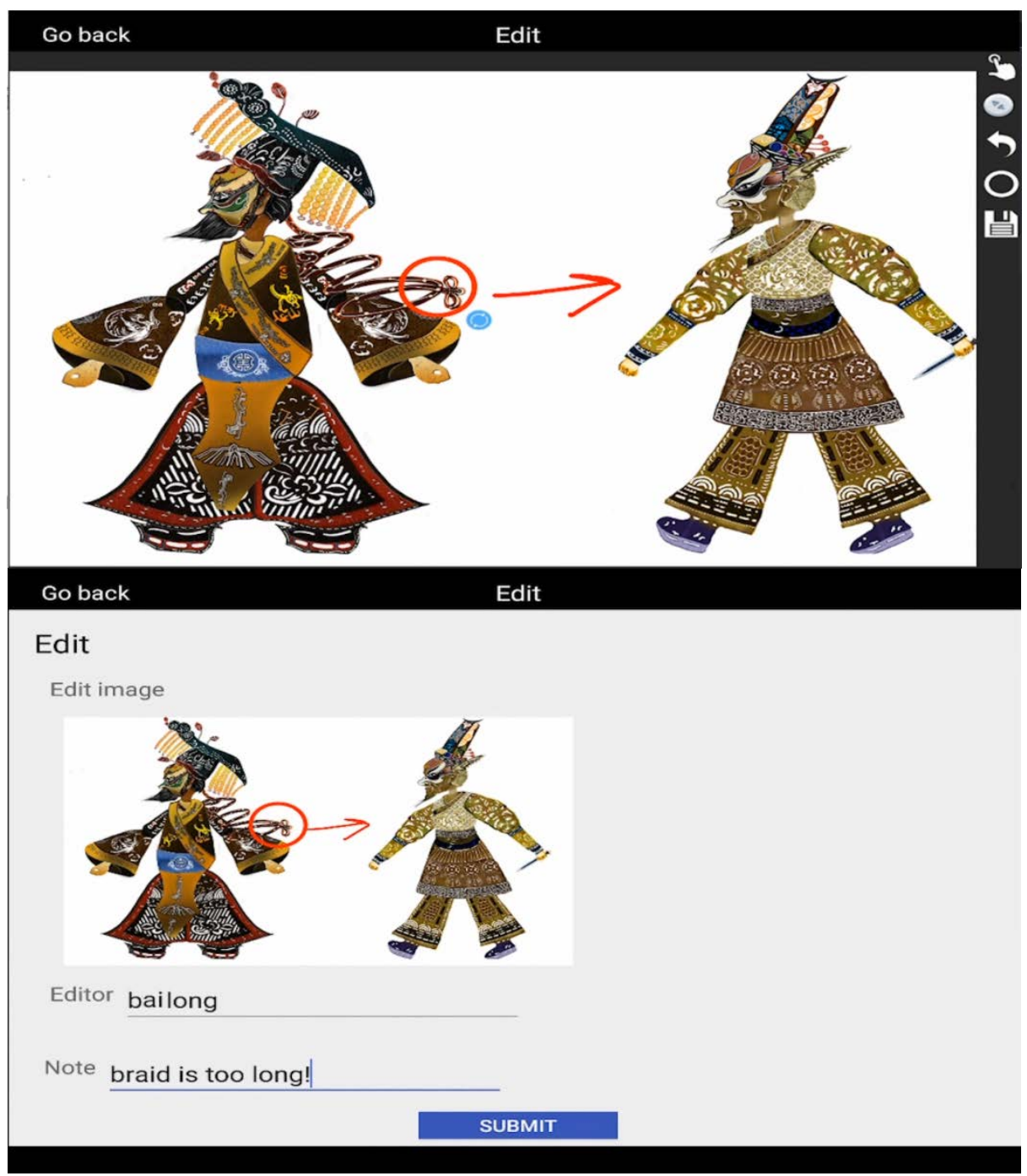

(a) Give cartoon design feedback 


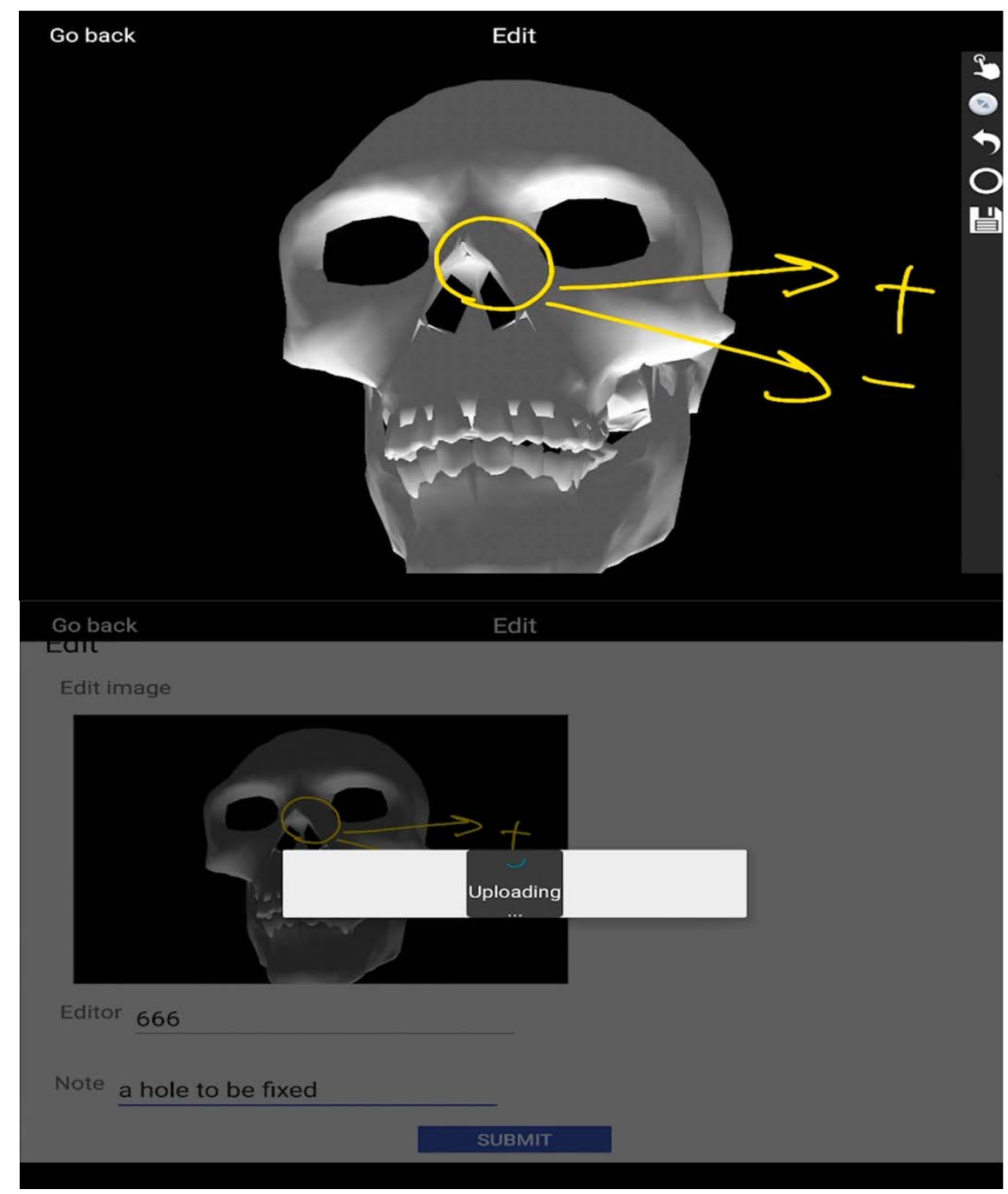

(b) Provide comments on 3D modeling

Fig.11. Screen shots of providing annotation for hyper type animation data 


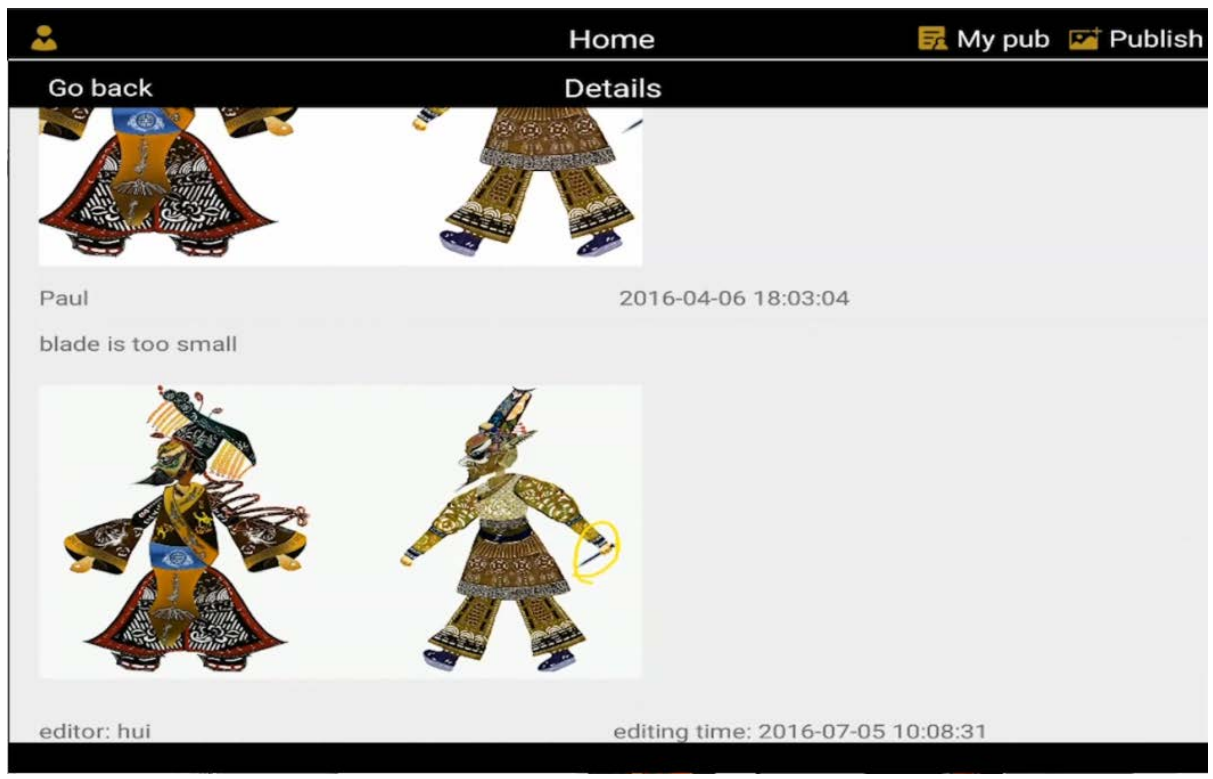

Fig.12. List of related asset versions and their notes

We built this App in Java for Android, which has been successfully tested and ran on Samsung Galaxy S2 tablet. We visited leading visual effects company Double Negative [28] and Picasso Pictures (one of London's top animation studios [29]) to conduct a questionnaire survey of industry experts. They provided positive feedback after preliminary trial.

\section{Conclusion}

The stage of our research is still proof-of-concept. We attempt to use semantic and oncological analysis to answer the emerging challenge of handling huge amounts of animation data produced in the industry and to find ways to address them. Constructed on an abstract high-level, the semantic model is proposed for animation asset to provide a systematic and standardized semantic description. Domain specified ontology has been developed in the context of traditional Chinese shadow puppetry play as the implementation of the proposed semantic model, which formalizes the construction of the animation data assets repository. And finally, a prototype of intelligent animation data management system is built upon a central repository developed on the base of the proposed ontology to facilitate the storage, organization, retrieval, utilization and reuse of animation assets.

Our main goal is to utilize semantic/ontology concept to improve the reusability, extensibility of the animation data assets and facilitate collaborative production. We hope this appealing semantic method may provide guidance for other researchers to 
define various domain specified ontologies and construct animation asset repositories depending on the context and application.

At the next step, the usability of the system will be examined carefully in our test. Animation artists and designers from the industry will be invited to participate. The evaluation of the semantic retrieval system will be also carried out to illustrate the benefit of this data management approach.

\section{Acknowledgment}

The research leading to these results has been partially supported by the People Programme (Marie Curie Actions) of the European Union's Seventh Framework Programme FP7/2007-2013/ under REA grant agreement $n^{\circ}$ [612627].

\section{References}

1. Liang, H., Sit, J., Chang, J., Zhang, J. J.: Computer animation data management: Review of evolution phases and emerging issues. Int J. Information Management 36(6): 1089-1100 (2016).

2. Desai, B. C.: The state of data. In Proceedings of the 18th international database engineering \& applications symposium, IDEAS’14, pp. 77-86 (2014).

3. Shotgunsoftware Homepage, http:// www.shotgunsoftware.com, last accessed 2017/5/21.

4. Alienbrain Homepage, http:// www.alienbrain.com, last accessed 2017/5/21.

5. Tactic Homepage, http:// www.tactic.net, last accessed 2017/5/21.

6. Fisher, M., Hanrahan, P.: Context-based search for 3D models. ACM Transactions on Graphics (TOG), 29(6), 182 (2010).

7. Mei, T., Rui, Y., Li, S., Tian, Q.: Multimedia search reranking: A literature survey. ACM Computing Surveys (CSUR), 46(3), 38 (2014).

8. Eitz, M., Richter, R., Boubekeur, T., Hildebrand, K., \& Alexa, M.: Sketch-based shape retrieval. ACM Trans Graph, 31(4), (1-10) (2012).

9. Tangelder, J. W. H., Veltkamp, R. C.: A survey of content based 3d shape retrieval methods. Multimedia Tools and Applications, 39(3), 441 (2008).

10. Li, z., Ramani, k.: Ontology-based design information extraction and retrieval. Artificial Intelligence for Engineering Design Analysis \& Manufacturing, 21(2), (137-154) (2007).

11. Kassimi, M. A., Elbeqqali, O.: Semantic based 3D model retrieval. International Conference on Multimedia Computing and Systems, pp.195-199). IEEE (2012).

12. Gruber, T.R.: A translation approach to portable ontology specifications, Knowledge acquisition, 5(2), 199-220 (1993).

13. Grüninger, M., Fox, M.S.: Methodology for the Design and Evaluation of Ontologies, (1995).

14. Borst, W. N.: Construction of engineering ontologies for knowledge sharing and reuse, Universiteit Twente (1997).

15. Studer, R., Benjamins, V. R., Fensel, D.: Knowledge engineering: principles and methods, Data \& knowledge engineering, 25(1), 161-197 (1998).

16. Falcidieno, B.: Aim@ shape project presentation, In Shape Modeling Applications, Proceedings, pp. 329, IEEE (2004). 
17. Funkhouser,T., Min, P., Kazhdan, M., Chen, J., Halderman, A., Dobkin D.,: A search engine for 3D models, ACM Transactions on Graphics (TOG) 22(1), 83-105 (2003).

18. Google 3D Warehouse, Available at: https://3dwarehouse.sketchup.com.

19. Ontology for Media Resources 1.0. Available at: http://www.w3.org/TR/mediaont-10.

20. Arndt, R., Troncy, R., Staab, S., Hardman, L.: Comm: A core ontology for multimediaannotation, In Handbook on Ontologies, pp. 403-421. Springer, Berlin Heidelberg (2009).

21. Thalmann, D., Farenc, N., Boulic, R.: Virtual human life simulation and database: why and how”, In Database Applications in Non-Traditional Environments (DANTE'99), Proceedings. 1999 International Symposium on, pp. 471-479, IEEE (1999).

22. De Boeck, J., Raymaekers, C., Coninx, K.: Comparing NiMMiT and data-driven notations for describing multimodal interaction, In Task Models and Diagrams for Users Interface Design, pp. 217-229. Springer, Berlin Heidelberg (2006).

23. Floty'nski, J., Walczak, K.: Conceptual semantic representation of 3d content, In Business Information Systems Workshops, pp. 244-257, Springer Berlin Heidelberg (2013).

24. Ruminski, D., Walczak, K.: Semantic contextual augmented reality environments, In Mixed and Augmented Reality (ISMAR), 2014 IEEE International Symposium on, pp. 401-404. IEEE (2014).

25. Teixeira, J.S.F., Sá, E.D.J.V., Fernandes, C. T.: A taxonomy of educational games compatible with the LOM-IEEE data model, Proceedings of Interdisciplinary Studies in Computer Science SCIENTIA, 44-59 (2008).

26. Dubin, D., Jett, J.: An Ontological Framework for Describing Games, In Proceedings of the 15th ACM/IEEE-CE on Joint Conference on Digital Libraries, pp. 165-168. ACM, (2015).

27. BinSubaih, A., Maddock,S., Romano, D.: Game logic portability, In Proceedings of the 2005 ACM SIGCHI International Conference on Advances in computer entertainment technology, pp. 458-461. ACM (2005).

28. Double Negative Homepage, http://www.dneg.com/, last accessed 2017/5/21.

29. Picasso Pictures Homepage, http:// www.picassopictures.com, last accessed 2017/5/21. 